Georgian Mathematical Journal

Volume 13 (2006), Number 1, 143-152

\title{
THE PSEUDO-SPECTRAL COLLOCATION METHOD FOR RESONANT LONG-SHORT NONLINEAR WAVE INTERACTION
}

\author{
ABDUR RASHID
}

\begin{abstract}
A pseudo-spectral collocation method for a class of equations describing resonant long-short wave interaction is studied. Semi-discrete and fully discrete Fourier pseudo-spectral collocation schemes are given. In fully discrete case we establish a three-level explicit scheme which is convenient and saves time in real computation. We use energy estimation methods to obtain error estimates for the approximate solutions.
\end{abstract}

2000 Mathematics Subject Classification: 65M70, 65M06, 65M12.

Key words and phrases: Long-short wave interaction, pseudo-spectral collocation method, energy estimation.

\section{INTRODUCTION}

Interaction phenomena between long waves and short waves have long been known and studied for many physical situations. This type of interaction is of interest in several fields of physics and fluid dynamics, e.g. water wave theory [5], electron-plasma/ion-field interaction [9] or diatomic lattice systems [13]. In the theory of capillary-gravity waves, Kawahara et al. [6] analyzed the coupled system

$$
\left\{\begin{array}{l}
i S_{t}+i c_{s} S_{x}+S_{x x}=\alpha L S, \\
L_{t}+c_{l} L_{x}+L_{x x x}+\left(L^{2}\right)_{x}+\beta|S|_{x}^{2}=0,
\end{array}\right.
$$

where $L$ and $S$ describe long and short water waves respectively, and $\alpha, \beta, c_{s}$ and $c_{l}$ are real constants. When the resonance condition $c_{s}=c_{l}$ holds, this equation is known as the coupled Schrödinger-KdV equation. The physical significance of (1) is that the dispersion of short waves is balanced by the nonlinear interaction of long waves with short waves, while the evolution of long waves is driven by the self-interaction of short waves.

One of the closely related resonant interactions is described by the system

$$
\left\{\begin{array}{l}
i \varepsilon_{t}+\varepsilon_{x x}=\alpha n \varepsilon, \quad t, x \in \mathbb{R}, \\
n_{t}+\beta|\varepsilon|_{x}^{2}=0, \\
\varepsilon(x, 0)=\varepsilon_{0}(x), n(x, 0)=n_{0}(x)
\end{array}\right.
$$

introduced by Benney [2] (see also [12], [5]). This system of equations has been studied using both inverse scattering methods ([12], [8]) and the theory of evolution equations ([1], [7], [11]). One important characteristic of Benney's equation (2) is that it is a completely integrable system. Moreover, Bekiranov 
et al. [1] showed that this system is well posed for weaker initial data, i.e. $\left(\varepsilon_{0}, n_{0}\right) \in H^{a}(\mathbb{R}) \times L^{1 / a}(\mathbb{R})$ for any $a>0$.

In this paper we consider a subclass of long-short wave interactions described by Benney's equation (2), namely the periodic initial boundary-value problem

$$
\left\{\begin{array}{l}
i \varepsilon_{t}+\varepsilon_{x x}=\alpha n \varepsilon, \quad x, t \in \mathbb{R}, \quad t>0, \\
n_{t}+\beta|\varepsilon|_{x}^{2}=0, \\
\varepsilon(x, 0)=\varepsilon_{0}(x), n(x, 0)=n_{0}(x), \\
\varepsilon(x+2 \pi, t)=\varepsilon(x, t), \quad n(x+2 \pi, t)=n(x, t) .
\end{array}\right.
$$

We investigate the implicit second order finite difference approximation in time, combined with pseudo-spectral collocation in space, for solving (3). Both the semi-discrete and the fully discrete schemes are analyzed and error estimation for both are found. The rate of convergence of the resulting schemes are $O\left(N^{-s}\right)$ and $O\left(\tau^{2}+N^{-s}\right)$ where $N$ is the number of spatial Fourier modes, $\tau$ is the discrete mesh spacing of the time variable $t$ and $s$ depends only on the smoothness of an exact solution.

For a further discussion, we introduce the following notation. Let $\Omega=[0,2 \pi]$ and $L^{2}(\Omega)$ denote the set of all square integrable functions with the inner product $(u, v)=\int_{0}^{2 \pi} u(x) v(x) d x$ and the norm $\|u\|^{2}=(u, u)$. Let $L^{\infty}(\Omega)$ denote the Lebesgue space with the norm $\|u\|_{L^{\infty}}=$ ess $\sup _{x \in \Omega}|u(x)|$ and $H_{p}^{s}(\Omega)$ denote the periodic Sobolev space with the norm $\|u\|_{s}=\left(\sum_{|\alpha| \leq s}\left\|D^{\alpha} u\right\|^{2}\right)^{1 / 2}$, we define

$$
\begin{aligned}
L^{2}\left(0, T ; H_{p}^{s}(\Omega)\right) & =\left\{u(\cdot, t) \in H_{p}^{s}(\Omega): \int_{0}^{T}\|u(\cdot, t)\|_{s}^{2} d t<\infty\right\}, \\
L^{\infty}\left(0, T ; H_{p}^{s}(\Omega)\right) & =\left\{u(\cdot, t) \in H_{p}^{s}(\Omega): \sup _{0 \leq t<T}\|u(\cdot, t)\|_{s} d t<\infty\right\} .
\end{aligned}
$$

Let $S_{N}=\operatorname{span}\left\{\psi_{k}=\frac{1}{\sqrt{2 \pi}} e^{i k x}:|k| \leq N\right\}$. Suppose $h=\frac{2 \pi}{2 N+1}$ is the mesh step of the variable $x$. The nodes are then $x_{\ell}=x_{0}+\ell h, x_{0}=-\pi, \ell=0,1, \ldots, 2 N$. The discrete inner product and norm in the interval $\Omega$ are defined by

$$
(u, v)=h \sum_{\ell=0}^{2 N} u\left(x_{\ell}\right) v\left(x_{\ell}\right), \quad\|u\|_{N}=(u, v)_{N}^{1 / 2} .
$$

Let $P_{N}: L^{2}(\Omega) \longrightarrow S_{N}$ be an orthogonal projection operator i.e.

$$
\left(P_{N} u, v\right)=(u, v), \quad \forall v \in S_{N} .
$$

and $P_{c}: C(\Omega) \longrightarrow S_{N}$ be an interpolation operator, i.e. such that for all $u \in C(\Omega)$

$$
P_{c} u\left(x_{\ell}\right)=u\left(x_{\ell}\right), \quad 0 \leq \ell \leq 2 N .
$$

For the discretization in the time variable $t$, let $\tau$ be the mesh spacing of $t$ and $R_{\tau}=\left\{t=k \tau: 0 \leq k \leq\left[\frac{T}{\tau}\right]\right\}$ and $u^{k}=u(x, k \tau)$. We define the following 
difference quotients as

$$
\begin{gathered}
u_{\widehat{t}}^{k}=\frac{1}{2 \tau}\left(u^{k+1}-u^{k-1}\right), \\
\widehat{u}^{k}=\frac{1}{2}\left(u^{k+1}+u^{k-1}\right) .
\end{gathered}
$$

\section{Some Lemmas}

In this section we state without proof a few lemmas which will be useful in the next section.

Lemma 1 ([3]). If $s \geq 0$ and $0 \leq \mu \leq s$, then for any $u \in H_{p}^{s}(\Omega)$

$$
\left\|u-P_{N} u\right\|_{\mu} \leq C N^{\mu-s}\|u\|_{s} .
$$

If, in addition, $s>1 / 2$, then

$$
\left\|u-P_{c} u\right\|_{\mu} \leq C N^{\mu-s}\|u\|_{s} \quad \text { and } \quad\left\|P_{c} u\right\|_{s} \leq C\|u\|_{s} .
$$

Lemma 2 ([10]). If $u, v \in C(\Omega)$, then

$$
\left(P_{c} u, P_{c} v\right)_{N}=\left(P_{c} u, P_{c} v\right)=(u, v)_{N} .
$$

Lemma 3 ([4]). If $s \geq 1$ and $u, v \in H^{s}(\Omega)$ then

$$
\|u v\|_{s} \leq C\|u\|_{s}\|v\|_{s} .
$$

Lemma 4 ([3]). Assume that the following conditions are fulfilled:

(i) $E(t)$ is a non-negative function defined on $R_{\tau}$.

(ii) $\rho, M$ and $c$ are non-negative constants.

(iii) For all $t \in R_{\tau}$ and $\max _{0 \leq t \leq T} E(t) \leq M$ we have

$$
E(t) \leq \rho+c \int_{0}^{t} E(\tau) d \tau .
$$

(iv) $E(0) \leq \rho \leq M e^{-c T}$.

Then for all $t \in R_{\tau}$ we have

$$
E(t) \leq \rho e^{c t}
$$

\section{The Semi-Discrete Pseudospectral Collocation Method}

The semi-discrete pseudospectral approximation of equation (3) consists in finding $\varepsilon_{c}, n_{c} \in S_{N}$ satisfying

$$
\left\{\begin{array}{l}
i \varepsilon_{c t}+\varepsilon_{c x x}-\alpha P_{c}\left(n_{c} \varepsilon_{c}\right)=0 \\
n_{c t}+\beta\left(P_{c}\left|\varepsilon_{c}\right|^{2}\right)_{x}=0 \\
\varepsilon_{c}(0)=P_{N} \varepsilon_{0}(x), n_{c}(0)=P_{N} n_{0}(x) .
\end{array}\right.
$$


Suppose that $(\varepsilon, n)$ is the solution of $(3)$ and $\left(\varepsilon_{c}, n_{c}\right)$ is the solution of (4). Setting

$$
\begin{aligned}
\varepsilon-\varepsilon_{c} & =\left(\varepsilon-P_{N} \varepsilon\right)+\left(P_{N} \varepsilon-\varepsilon_{c}\right)=\lambda+\xi, \\
n-n_{c} & =\left(n-P_{N} n\right)+\left(P_{N} n-n_{c}\right)=\sigma+\theta,
\end{aligned}
$$

one sees that, by (3) and (4), $\xi$ and $\theta$ satisfy the system

$$
\left\{\begin{array}{l}
\left.i\left(\xi_{t}, w\right)-\left(\xi_{x}, w_{x}\right)-\alpha\left(\left(I-P_{c}\right)(n \varepsilon), w\right)+\alpha\left(P_{c}\left(n_{c} \varepsilon_{c}-n \varepsilon\right), w\right)\right)=0, \\
\left(\theta_{t}, w\right)-\beta\left(\left(I-P_{c}\right)|\varepsilon|^{2}, w_{x}\right)+\beta\left(P_{c}\left(\left|\varepsilon_{c}\right|^{2}-|\varepsilon|^{2}\right), w_{x}\right)=0 .
\end{array}\right.
$$

Setting $w=\xi$ in the first equation of (5), we have

$$
\frac{1}{2} \frac{d}{d t}\|\xi\|^{2}=\alpha I_{m}\left(\left(I-P_{c}\right)(n \varepsilon), \xi\right)+\alpha I_{m}\left(P_{c}\left(n \varepsilon-n_{c} \varepsilon_{c}\right), \xi\right),
$$

and

$$
\left|\alpha I_{m}\left(\left(I-P_{c}\right)(n \varepsilon), \xi\right)\right| \leq|\alpha|\left(\left\|\left(I-P_{c}\right)(n \varepsilon)\right\|^{2}+\|\xi\|^{2}\right) .
$$

But by Lemmas 1 and 3 we obtain

$$
\left\|\left(I-P_{c}\right)(n \varepsilon)\right\| \leq C N^{-s}\|n \varepsilon\|_{s} \leq C N^{-s}\|n\|_{s}\|\varepsilon\|_{s}
$$

and

$$
\left|\alpha I_{m}\left(\left(I-P_{c}\right)(n \varepsilon), \xi\right)\right| \leq C\left(\|\xi\|^{2}+N^{-2 s}\right),
$$

where $C=C\left(\alpha,\|n\|_{s},\|\varepsilon\|_{s}\right)$ and so

$$
\left.\left|\alpha I_{m}\left(P_{c}\left(n \varepsilon-n_{c} \varepsilon_{c}\right), \xi\right)\right| \leq|\alpha|\left(\left\|P_{c}\left(n \varepsilon-n_{c} \varepsilon_{c}\right)\right\|^{2}+\| \xi\right) \|^{2}\right) .
$$

But

$$
\begin{aligned}
\left\|P_{c}\left(n \varepsilon-n_{c} \varepsilon_{c}\right)\right\| & =\left\|P_{c}\left(n\left(\varepsilon-\varepsilon_{c}\right)\right)\right\|+\left\|P_{c}\left(\varepsilon_{c}\left(n-n_{c}\right)\right)\right\| \\
& \leq\|n\|_{\infty}\left\|P_{c}\left(\varepsilon-\varepsilon_{c}\right)\right\|+\left\|\varepsilon_{c}\right\|_{\infty}\left\|P_{c}\left(n-n_{c}\right)\right\| .
\end{aligned}
$$

Since $P_{c}\left(u-u_{c}\right)=P_{c} u-u_{c}=-\left(I-P_{c}\right) u+\left(I-P_{N}\right) u+\left(P_{N} u-u\right)$, by Lemma 1 , we have the following results:

$$
\begin{aligned}
\left\|P_{c}\left(\varepsilon-\varepsilon_{c}\right)\right\| & \leq C N^{-s}\|\varepsilon\|_{s}+\|\xi\|, \\
\left\|P_{c}\left(n-n_{c}\right)\right\| & \leq C N^{-s}\|n\|_{s}+\|\theta\| .
\end{aligned}
$$

Therefore

$$
\left|\alpha I_{m}\left(P_{c}\left(n \varepsilon-n_{c} \varepsilon_{c}\right), \xi\right)\right| \leq C\left(\|\xi\|^{2}+\|\theta\|^{2}+N^{-2 s}\right),
$$

and substituting (8) and (7) into (6), we have

$$
\frac{1}{2} \frac{d}{d t}\|\xi\|^{2} \leq C\left(\|\xi\|^{2}+\|\theta\|^{2}+N^{-2 s}\right),
$$

where $C=C\left(\alpha,\|n\|_{\infty},\|\varepsilon\|_{\infty},\|n\|_{s},\|\varepsilon\|_{s}\right)$.

Setting $w=\xi_{t}$ in the first equation of (5), we have

$$
\frac{1}{2} \frac{d}{d t}\left\|\xi_{x}\right\|^{2}=\alpha \operatorname{Re}\left(\left(I-P_{c}\right)(n \varepsilon), \xi_{t}\right)+\alpha \operatorname{Re}\left(P_{c}\left(n \varepsilon-n_{c} \varepsilon_{c}\right), \xi_{t}\right) .
$$

Similarly to the proof of (9), we get

$$
\frac{1}{2} \frac{d}{d t}\left\|\xi_{x}\right\|^{2} \leq C\left(\left\|\xi_{t}\right\|^{2}+\|\xi\|^{2}+\|\theta\|^{2}+N^{-2 s}\right)
$$


Differentiate the first equation of (5) with respect to $t$ and take $w=\xi_{t}$ to obtain

$$
\frac{1}{2} \frac{d}{d t}\left\|\xi_{t}\right\|^{2}=\alpha I_{m}\left(\left(I-P_{c}\right)(n \varepsilon)_{t}, \xi_{t}\right)+\alpha I_{m}\left(P_{c}\left(n \varepsilon-n_{c} \varepsilon_{c}\right)_{t}, \xi_{t}\right),
$$

and since

$$
\left(n \varepsilon-n_{c} \varepsilon_{c}\right)_{t}=\varepsilon_{c t}\left(n-n_{c}\right)+\varepsilon_{c}\left(n-n_{c t}\right)+n_{t}\left(\varepsilon-\varepsilon_{c}\right)+n\left(\varepsilon_{t}-\varepsilon_{c t}\right),
$$

we have

$$
\begin{aligned}
\left|\alpha I_{m}\left(P_{c}\left(n \varepsilon-n_{c} \varepsilon_{c}\right)_{t}, \xi_{t}\right)\right| \leq & |\alpha|\left\|\xi_{t}\right\|\left(\left\|P_{N} \varepsilon_{t}\right\|_{\infty}\left\|P_{c}\left(n-n_{c}\right)\right\|+\left\|\varepsilon_{c}\right\|_{\infty}\left\|P_{c}\left(n-n_{c t}\right)\right\|\right. \\
& \left.+\left\|n_{t}\right\|_{\infty}\left\|P_{c}\left(\varepsilon-\varepsilon_{c}\right)\right\|+\|n\|_{\infty}\left\|P_{c}\left(\varepsilon_{t}-\varepsilon_{c t}\right)\right\|\right) .
\end{aligned}
$$

By Lemmas 1 and 3, we have

$$
\left|\alpha I_{m}\left(P_{c}\left(n \varepsilon-n_{c} \varepsilon_{c}\right)_{t}, \xi_{t}\right)\right| \leq C\left(\left\|\xi_{t}\right\|^{2}+\|\theta\|^{2}+\left\|\theta_{t}\right\|^{2}+\|\xi\|^{2}+N^{-2 s}\right)
$$

and

$$
\left|\alpha I_{m}\left(\left(I-P_{c}\right)(n \varepsilon)_{t}, \xi_{t}\right)\right| \leq C\left(\left\|\xi_{t}\right\|^{2}+N^{-2 s}\left\|n_{t}\right\|_{s}\left\|\varepsilon_{t}\right\|_{s}\right) .
$$

Putting (13) and (14) in (12), we get

$$
\frac{1}{2} \frac{d}{d t}\left\|\xi_{t}\right\|^{2} \leq C\left(\left\|\xi_{t}\right\|^{2}+\|\xi\|_{1}^{2}+\|\theta\|^{2}+\left\|\theta_{t}\right\|^{2}+N^{-2 s}\right)
$$

where $C=C\left(\alpha,\left\|\varepsilon_{c}\right\|_{\infty},\left\|n_{t}\right\|_{\infty},\|n\|_{\infty},\left\|\varepsilon_{t}\right\|,\|\varepsilon\|_{\infty},\left\|\varepsilon_{c t}\right\|,\left\|n_{t}\right\|_{s},\|n\|_{s},\left\|\varepsilon_{t}\right\|_{s}\right)$. Setting $w=\theta$ in the second equation of (5), we get

$$
\left(\theta_{t}, \theta\right)-\beta\left(\left(I-P_{c}\right)|\varepsilon|^{2}, \theta_{x}\right)+\beta\left(P_{c}\left(\left|\varepsilon_{c}\right|^{2}-|\varepsilon|^{2}\right), \theta_{x}\right)=0
$$

and so

$$
\frac{1}{2} \frac{d}{d t}\|\theta\|^{2}=\beta\left(\left(I-P_{c}\right)|\varepsilon|^{2}, \theta_{x}\right)+\beta\left(P_{c}\left(|\varepsilon|^{2}-\left|\varepsilon_{c}\right|^{2}\right), \theta_{x}\right) .
$$

To estimate the right-hand side of equation (16), we consider

$$
\begin{aligned}
\left|\beta\left(\left(I-P_{c}\right)|\varepsilon|^{2}, \theta_{x}\right)\right| & \leq|\beta|\left\|\left(I-P_{c}\right)|\varepsilon|^{2}\right\|\left\|\theta_{x}\right\| \\
& \leq C\left(\left\|\theta_{x}\right\|^{2}+\left\|\left(I-P_{c}\right)|\varepsilon|^{2}\right\|^{2}\right) \leq C\left(\left\|\theta_{x}\right\|^{2}+N^{-2 s}\right)
\end{aligned}
$$

and

$$
\left|\alpha\left(P_{c}\left(|\varepsilon|^{2}-\left|\varepsilon_{c}\right|^{2}\right), \theta_{x}\right)\right| \leq C\left(\left\|P_{c}\left(|\varepsilon|^{2}-\left|\varepsilon_{c}\right|^{2}\right)\right\|^{2}+\left\|\theta_{x}\right\|^{2}\right),
$$

where

$$
\begin{aligned}
\left\|P_{c}\left(|\varepsilon|^{2}-\left|\varepsilon_{c}\right|^{2}\right)\right\| & =\left\|P_{c}\left(\varepsilon \bar{\varepsilon}-\varepsilon_{c} \overline{\varepsilon_{c}}\right)\right\| \\
& =\left\|P_{c}\left(\varepsilon\left(\bar{\varepsilon}-\varepsilon_{c}\right)\right)+P_{c}\left(\bar{\varepsilon}\left(\varepsilon-\varepsilon_{c}\right)\right)\right\| \\
& \leq\|\varepsilon\|_{\infty}\left\|P_{c}\left(\overline{\varepsilon-\varepsilon_{c}}\right)\right\|+\left\|\overline{\varepsilon_{c}}\right\|_{\infty}\left\|P_{c}\left(\varepsilon-\varepsilon_{c}\right)\right\| \\
& \leq\left(\|\varepsilon\|_{\infty}+\left\|\varepsilon_{c}\right\|_{\infty}\right)\left\|P_{c}\left(\varepsilon-\varepsilon_{c}\right)\right\| \\
& \leq\left(\|\varepsilon\|_{\infty}+\left\|\varepsilon_{c}\right\|_{\infty}\right)\left(C N^{-s}\|\varepsilon\|_{s}+\|\xi\|\right) .
\end{aligned}
$$

Substituting estimates (17) and (18) into (16), we get

$$
\frac{1}{2} \frac{d}{d t}\|\theta\|^{2} \leq C\left(\left\|\theta_{x}\right\|^{2}+\|\xi\|^{2}+C N^{-2 s}\right) .
$$


Combining (9), (11), (15) and (19) we find

$$
\frac{d}{d t}\left(\|\xi\|^{2}+\|\xi\|_{1}^{2}+\left\|\xi_{t}\right\|^{2}+\|\theta\|^{2}\right) \leq C\left(\|\xi\|^{2}+\|\xi\|_{1}^{2}+\left\|\xi_{t}\right\|^{2}+\|\theta\|^{2}+N^{-2 s}\right) .
$$

By applying Gronwall's inequality we obtain

$$
\begin{aligned}
\|\xi(t)\|^{2} & +\|\xi(t)\|_{1}^{2}+\left\|\xi_{t}(t)\right\|^{2}+\|\theta(t)\|^{2} \\
& \leq\|\xi(0)\|^{2}+\|\xi(0)\|_{1}^{2}+\left\|\xi_{t}(0)\right\|^{2}+\|\theta(0)\|^{2}+C N^{-2 s} \\
& +C \int_{0}^{t}\left(\|\xi(\tau)\|^{2}+\|\xi(\tau)\|_{1}^{2}+\left\|\xi_{t}(\tau)\right\|^{2}+\|\theta(\tau)\|^{2}\right) d \tau .
\end{aligned}
$$

The initial conditions read as

$$
\xi_{c}(0)=\theta_{c}(0)=0, \quad \xi_{x}(0)=0 .
$$

At $t=0$, setting $w=\xi_{t}(0)$ in $(5)$, we have

$$
\left\|\xi_{t}(0)\right\|^{2} \leq C\left(\left\|\left(I-P_{c}\right)\left(n_{0} \varepsilon_{0}\right)\right\|^{2}+\left\|P_{c}\left(P_{N} n_{0} P_{N} \varepsilon_{0}-n_{0} \varepsilon_{0}\right)\right\|^{2},\right.
$$

and

$$
\left\|\xi_{t}(0)\right\|^{2} \leq C N^{-2 s}
$$

Let

$$
E(t)=\|\xi(t)\|^{2}+\|\xi(t)\|_{1}^{2}+\left\|\xi_{t}(t)\right\|^{2}+\|\theta(t)\|^{2} .
$$

Using (21) and (22) in (20), we get

$$
E(t) \leq C N^{-2 s}+C \int_{0}^{t} E(\tau) d \tau .
$$

Thus we have proved

Theorem 1. Suppose $\varepsilon$ and $n$ are solutions of equation (3) and assume $\varepsilon \in L^{\infty}\left(0, T ; H_{p}^{s+1}\right), \varepsilon_{t}, n, n_{t} \in L^{\infty}\left(0, T ; H_{p}^{s}\right)$. Then for $\varepsilon_{c}$ and $n_{c}$ the solutions for the pseudo-spectral scheme (4), there exist positive constants $M$ and $C$ such that $N \geq M$ and $s \geq 2$,

$$
\left\|\varepsilon(t)-\varepsilon_{c}(t)\right\|_{1}+\left\|\varepsilon_{t}(t)-\varepsilon_{c t}(t)\right\|_{1}+\left\|n(t)-n_{c}(t)\right\| \leq C N^{-2 s}
$$

where $C$ is independent of $N$.

\section{The Fully Discrete Pseudospectral Collocation Method}

We consider the fully discrete pseudospectral collocation method which consists in finding $\varepsilon_{c}^{k}, n_{c}^{k} \in S_{N}$ such that for $k=1, \ldots,\left[\frac{T}{\tau}\right]$ the equations

$$
\left\{\begin{array}{l}
i \varepsilon_{c \widehat{t}}^{k}+\widehat{\varepsilon}_{c x x}^{k}-\alpha P_{c}\left(n_{c}^{k} \varepsilon_{c}^{k}\right)=0, \\
n_{c \widehat{t}}+\beta\left(P_{c}\left|\varepsilon_{c}^{k}\right|^{2}\right)_{x}=0, \\
\varepsilon_{c}^{0}=P_{N} \varepsilon_{0}, n_{c}^{0}=P_{N} n_{0}, \varepsilon_{c}^{1}=P_{N} \varepsilon_{1}(x), n_{c}^{1}=P_{N} n_{1}
\end{array}\right.
$$

are satisfied at $x=x_{j}, j=0, \ldots, 2 N$. 
Let

$$
\begin{aligned}
\varepsilon^{k}-\varepsilon_{c}^{k}=\left(\varepsilon^{k}-P_{N} \varepsilon^{k}\right)+\left(P_{N} \varepsilon^{k}-\varepsilon_{c}^{k}\right) & =\lambda^{k}+\xi^{k}, \\
n^{k}-n_{c}^{k}=\left(n^{k}-P_{N} n^{k}\right)+\left(P_{N} n^{k}-n_{c}^{k}\right) & =\sigma^{k}+\theta^{k} .
\end{aligned}
$$

From equations (3) and (23), we get

$$
\left\{\begin{array}{l}
i\left(\xi_{\widehat{t}}^{k}, w\right)-\left(\widehat{\xi}_{x}^{k}, w\right)-\alpha\left(P_{c}\left(n^{k} \widehat{\varepsilon}^{k}-n_{c}^{k} \widehat{\varepsilon}_{c}^{k}\right), w\right)=\left(G_{1}^{k}, w\right), \\
\left(\theta_{\widehat{t}}, w\right)-\beta\left(P_{c}\left(\left|\varepsilon^{k}\right|^{2}-\left|\varepsilon_{c}^{k}\right|^{2}\right), w_{x}\right)=\left(G_{2}^{k}, w\right)+\left(G_{3}^{k}, w_{x}\right),
\end{array}\right.
$$

where

$$
\begin{aligned}
G_{1}^{k}= & i\left(\varepsilon_{\widehat{t}}^{k}-\varepsilon_{t}^{k}\right)+\left(\widehat{\varepsilon}_{x x}^{k}-\varepsilon_{x x}^{k}\right)+\alpha\left(I-P_{c}\right)\left(n^{k} \widehat{\varepsilon}^{k}\right)+\alpha n^{k}\left(\varepsilon^{k}-\widehat{\varepsilon}^{k}\right) \\
= & \frac{i \tau^{2}}{12}\left[\frac{\partial^{3}}{\partial t^{3}} \varepsilon\left(t_{1}^{k}\right)+\frac{\partial^{3}}{\partial t^{3}} \varepsilon\left(t_{2}^{k}\right)\right]-\frac{\tau^{2}}{2}\left[\frac{\partial^{2}}{\partial t^{2}} \varepsilon_{x x}\left(t_{3}^{k}\right)+\frac{\partial^{2}}{\partial t^{2}} \varepsilon_{x x}\left(t_{4}^{k}\right)\right] \\
& +\frac{-\alpha n^{k} \tau^{2}}{4}\left[\frac{\partial^{3}}{\partial t^{3}} \varepsilon\left(t_{5}^{k}\right)+\frac{\partial^{3}}{\partial t^{3}} \varepsilon\left(t_{6}^{k}\right)\right]+\alpha\left(I-P_{c}\right)\left(n^{k} \widehat{\varepsilon}^{K}\right), \\
G_{2}^{k}= & \left(n_{\widehat{t}}^{k}-n_{t}^{k}\right)=\frac{\tau^{2}}{12}\left[\frac{\partial^{3}}{\partial t^{3}} \varepsilon\left(t_{7}^{k}\right)+\frac{\partial^{3}}{\partial t^{3}} \varepsilon\left(t_{8}^{k}\right)\right], \\
G_{3}^{k}= & -\beta\left(I-P_{c}\right)\left|\varepsilon^{k}\right|^{2} .
\end{aligned}
$$

Setting $w=\widehat{\xi}^{k}$ in the first equation of (24), we get

$$
\frac{1}{4 \tau}\left[\left\|\xi^{k+1}\right\|^{2}-\left\|\xi^{k-1}\right\|^{2}\right]=\alpha I_{m}\left(P_{c}\left(n^{k} \widehat{\varepsilon}^{k}-n_{c}^{k} \widehat{\varepsilon}_{c}^{k}\right), \widehat{\xi}^{k}\right)+I_{m}\left(G_{1}^{k}, \widehat{\xi}^{k}\right) .
$$

Since we have

$$
\begin{gathered}
\left|\alpha I_{m}\left(P_{c}\left(n^{k} \widehat{\varepsilon}^{k}-n_{c}^{k} \widehat{\varepsilon}_{c}^{k}\right), \widehat{\xi}^{k}\right)\right| \leq C\left(\left\|\widehat{\xi}^{k}\right\|^{2}+\left\|\theta^{k}\right\|^{2}+\tau^{4}+N^{-2 s}\right), \\
\left|I_{m}\left(G_{1}^{k}, \widehat{\xi}^{k}\right)\right| \leq C\left(\left\|\widehat{\xi}^{k}\right\|^{2}+\tau^{4}+N^{-2 s}\right),
\end{gathered}
$$

putting the above estimate (26) into (25), we get

$$
\frac{1}{4 \tau}\left[\left\|\xi^{k+1}\right\|^{2}-\left\|\xi^{k-1}\right\|^{2}\right] \leq C\left(\left\|\widehat{\xi}^{k}\right\|^{2}+\left\|\theta^{k}\right\|^{2}+\tau^{4}+N^{-2 s}\right) .
$$

By summing (27) w.r.t. $k=1,2, \ldots, n$ we find

$$
\left\|\xi^{n+1}\right\|^{2} \leq\left\|\xi^{0}\right\|^{2}+\left\|\xi^{1}\right\|^{2}+c\left(\tau^{4}+N^{-2 s}\right)+C \tau \sum_{k=1}^{n}\left(\left\|\widehat{\xi}^{k}\right\|^{2}+\left\|\theta^{k}\right\|^{2}\right) .
$$

Setting $w=\xi_{\widehat{t}}^{k}$ in the first equation of (24), we get

$$
\frac{1}{4 \tau}\left[\left\|\xi_{x}^{k+1}\right\|^{2}-\left\|\xi_{x}^{k-1}\right\|^{2}\right]=-\alpha \operatorname{Re}\left(P_{c}\left(n^{k} \widehat{\varepsilon}^{k}-n_{c}^{k} \widehat{\varepsilon}_{c}^{k}\right), \xi_{\widehat{t}}^{k}\right)+\operatorname{Re}\left(G_{1}^{k}, \xi_{\widehat{t}}^{k}\right)
$$

and hence

$$
\frac{1}{4 \tau}\left[\left\|\xi_{x}^{k+1}\right\|^{2}-\left\|\xi_{x}^{k-1}\right\|^{2}\right] \leq C\left(\left\|\xi_{\widehat{t}}^{k}\right\|^{2}+\left\|\widehat{\xi}^{k}\right\|^{2}+\left\|\theta^{k}\right\|^{2}+\tau^{4}+N^{-2 s}\right) .
$$


By summing (29) w.r.t. $k=1,2, \ldots, n$ we find

$$
\begin{aligned}
\left\|\xi_{x}^{n+1}\right\|^{2} \leq & \left\|\xi_{x}^{0}\right\|^{2}+\left\|\xi_{x}^{1}\right\|^{2}+C\left(\tau^{4}+N^{-2 s}\right) \\
& +C \tau \sum_{k=1}^{n}\left(\left\|\xi_{\widehat{t}}^{k}\right\|^{2}+\left\|\widehat{\xi}^{k}\right\|^{2}+\left\|\theta^{k}\right\|^{2}\right) .
\end{aligned}
$$

Differentiating the first equation of (24) with respect to $t$ and taking $w=\widehat{\xi}_{\widehat{t}}^{k}$, we get

$$
\frac{1}{4 \tau}\left[\left\|\xi_{\widehat{t}}^{k+1}\right\|^{2}-\left\|\xi_{\widehat{t}}^{k-1}\right\|^{2}\right]=\alpha I_{m}\left(P_{c}\left(n^{k} \widehat{\varepsilon}^{k}-n_{c}^{k} \widehat{\varepsilon}_{c}^{k}\right)_{t}, \widehat{\xi}_{\widehat{t}}^{k}\right)+I_{m}\left(G_{1 t}^{k}, \widehat{\xi}_{\widehat{t}}^{k}\right) .
$$

Substituting the estimates

$$
\begin{gathered}
\left|\alpha I_{m}\left(P_{c}\left(n^{k} \widehat{\varepsilon}^{k}-n_{c}^{k} \widehat{\varepsilon}_{c}^{k}\right)_{t}, \widehat{\xi}_{\widehat{t}}^{k}\right)\right| \leq C\left(\left\|\widehat{\xi}_{\widehat{t}}^{k}\right\|^{2}+\left\|\theta^{k+1}\right\|^{2}+\left\|\theta_{\widehat{t}}^{k}\right\|^{2}+\left\|\widehat{\xi}^{k+1}\right\|^{2}+N^{-2 s}\right), \\
\left|I_{m}\left(G_{1 t}^{k}, \widehat{\xi}_{\widehat{t}}^{k}\right)\right| \leq C\left(\left\|\widehat{\xi}_{\widehat{t}}^{k}\right\|^{2}+\tau^{4}+N^{-2 s}\right),
\end{gathered}
$$

into (31) and summing from $k=1$ to $n$, we get

$$
\begin{aligned}
\left\|\xi_{\widehat{t}}^{n}\right\|^{2} \leq & \left\|\xi_{\widehat{t}}^{0}\right\|^{2}+\left\|\xi_{\widehat{t}}^{1}\right\|^{2}+C\left(\tau^{4}+N^{-2 s}\right) \\
& +C \tau \sum_{k=1}^{n}\left(\left\|\widehat{\xi}_{\widehat{t}}^{k}\right\|^{2}+\left\|\widehat{\xi}^{k}\right\|^{2}+\left\|\theta^{k+1}\right\|^{2}+\left\|\theta_{\widehat{t}}^{k}\right\|^{2}\right) .
\end{aligned}
$$

Setting $w=\theta^{k}$ in the second equation of (24), we get

$$
\frac{1}{4 \tau}\left[\left\|\theta^{k+1}\right\|^{2}-\left\|\theta^{k-1}\right\|^{2}\right]=\beta\left(P_{c}\left(\left|\varepsilon^{k}\right|^{2}-\left|\varepsilon_{c}^{k}\right|^{2}\right), \widehat{\theta}_{x}^{k}\right)+\left(G_{2}^{k}, \widehat{\theta}^{k}\right)+\left(G_{3}^{k}, \widehat{\theta}_{x}^{k}\right) .
$$

To estimate the right-hand side of (33) notice that the following inequalities hold

$$
\begin{aligned}
\left|\beta\left(P_{c}\left(\left|\varepsilon^{k}\right|^{2}-\left|\varepsilon_{c}^{k}\right|^{2}\right), \widehat{\theta}_{x}^{k}\right)\right| \leq C\left(\left\|\widehat{\theta}_{x}^{k}\right\|^{2}+\left\|\xi^{k}\right\|^{2}+N^{-2 s}\right), \\
\left|\left(G_{2}^{k}, \widehat{\theta}^{k}\right)\right| \leq C\left(\left\|\widehat{\theta}^{k}\right\|^{2}+\tau^{4}\right), \\
\left|\left(G_{3}^{k}, \widehat{\theta}_{x}^{k}\right)\right| \leq C\left(\left\|\widehat{\theta}^{k}\right\|^{2}+N^{-2 s}\right),
\end{aligned}
$$

and hence

$$
\frac{1}{4 \tau}\left[\left\|\theta^{k+1}\right\|^{2}-\left\|\theta^{k-1}\right\|^{2}\right] \leq C\left(\left\|\widehat{\theta}_{x}^{k}\right\|^{2}+\left\|\theta^{k}\right\|^{2}+\left\|\xi^{k}\right\|^{2}+\tau^{4}+N^{-2 s}\right) .
$$

By summing (34) w.r.t. $k=1,2, \ldots, n$ we get

$$
\begin{aligned}
\left\|\theta^{n+1}\right\|^{2} \leq & \left\|\theta^{0}\right\|^{2}+\left\|\theta^{1}\right\|^{2}+C\left(\tau^{4}+N^{-2 s}\right) \\
& +C \tau \sum_{k=1}^{n}\left(\left\|\widehat{\theta}_{x}^{k}\right\|^{2}+\left\|\theta^{k}\right\|^{2}+\left\|\xi^{k}\right\|^{2}\right) .
\end{aligned}
$$

Setting $w=\theta_{\widehat{t}}^{k}$ in the second equation of (24) we find

$$
\left\|\theta_{\widehat{t}}^{k}\right\|^{2} \leq C\left(\left\|\theta_{\widehat{t} x}^{k}\right\|^{2}+\left\|\theta_{\widehat{t}}^{k}\right\|^{2}+\left\|\xi^{k}\right\|^{2}+\tau^{4}+N^{-2 s}\right) .
$$


Combining (28), (30), (32) and (35), we get

$$
\begin{aligned}
E^{N}= & \left\|\xi^{n+1}\right\|_{1}^{2}+\left\|\theta^{n+1}\right\|^{2}+\left\|\xi_{\widehat{t}}^{n}\right\|^{2} \\
\leq & \left(\left\|\xi^{1}\right\|_{1}^{2}+\left\|\theta^{1}\right\|^{2}+\left\|\xi_{\widehat{t}}^{1}\right\|^{2}+\left\|\theta^{0}\right\|^{2}+\tau^{4}+N^{-2 s}\right) \\
& +C \tau \sum_{k=1}^{n}\left(\left\|\widehat{\xi}^{k}\right\|^{2}+\left\|\xi_{\widehat{t}}^{k}\right\|^{2}+\left\|\widehat{\xi}_{\widehat{t}}^{k}\right\|^{2}+\left\|\theta^{k}\right\|^{2}+\left\|\widehat{\theta}^{k}\right\|^{2}\right),
\end{aligned}
$$

and hence

$$
E^{N} \leq C\left(\left\|\xi^{1}\right\|_{1}^{2}+\left\|\theta^{1}\right\|^{2}+\left\|\xi_{\hat{t}}^{1}\right\|^{2}+\left\|\theta^{0}\right\|^{2}+\tau^{4}+N^{-2 s}\right)+C \tau \sum_{k=0}^{n} E^{k-1} .
$$

Using the initial conditions $\xi^{0}=0$ and $\xi_{x}^{0}=0$, substituting $k=1$ into the first equation of (24) and setting $w=\xi_{\widehat{t}}^{1}$, we find

$$
\left\|\xi_{\widehat{t}}^{1}\right\|^{2} \leq C\left(\left\|\xi^{2}\right\|^{2}+\left\|\xi^{1}\right\|^{2}+\left\|\theta^{1}\right\|^{2}+\tau^{4}+N^{-2 s}\right) .
$$

Again taking $k=1$ in the first equation of (24) and setting $w=\widehat{\xi}^{1}$ we get

$$
\left\|\xi^{2}\right\|^{2} \leq C\left(\left\|\xi^{1}\right\|^{2}+\left\|\theta^{1}\right\|^{2}+\tau^{4}+N^{-2 s}\right)
$$

and since

$$
\left\|\xi^{1}\right\|_{1}^{2}+\left\|\theta^{1}\right\|^{2} \leq C\left(\tau^{4}+N^{-2 s}\right)
$$

equation (36) can be rewritten as

$$
E^{N}(t) \leq C\left(\tau^{4}+N^{-2 s}\right)+C \tau \sum_{k=0}^{n} E^{k-1}
$$

By applying Lemma 4 we obtain

$$
C\left(\tau^{4}+N^{-2 s}\right) \leq M e^{-c T},
$$

and so the estimate for $E^{N}(t)$ in (37) takes the form

$$
E^{N}(t) \leq C\left(\tau^{4}+N^{-2 s}\right) e^{c(n+1) \tau}, \quad \forall(n+1) \tau \leq T .
$$

Thus we have proved

Theorem 2. Assume that $\varepsilon(x, t) \in L^{\infty}\left(0, T ; H_{p}^{s+1}\right), \varepsilon_{t}, n, n_{t} \in L^{\infty}\left(0, T ; H_{p}^{s}\right)$, $\varepsilon_{t t}, n_{t t}, \in L^{\infty}\left(0, T ; H_{p}^{1}\right), \varepsilon_{t t t}, n_{t t t}, \in L^{\infty}\left(0, T ; H_{p}^{0}\right)$. Then there exist constants $C$ and $\delta$ such that $\tau^{2}+N^{-s}<\delta$ and $\forall n,(n+1) \tau \leq t$,

$$
\left\|\varepsilon^{n+1}-\varepsilon_{c}^{n+1}\right\|_{1}+\left\|\varepsilon_{\hat{t}}^{n}-\varepsilon_{c \hat{t}}^{n}\right\|_{1}+\left\|n^{n+1}-n_{c}^{n+1}\right\| \leq C\left(\tau^{2}+N^{-s}\right) .
$$




\section{REFERENCES}

1. D. Bekiranov, T. Ogawa and G. Ponce, On the well-posedness of Benney's interaction equation of short and long waves. Adv. Differential Equations 1(1996), No. 6, 919-937.

2. D. J. Benney, A general theory for interactions between short and long waves. Studies in Appl. Math. 56(1976/77), No. 1, 81-94.

3. C. Canuto, M. Y. Hussaini, A. Quarteroni and T. A. Zang, Spectral methods in fluid dynamics. Springer Series in Computational Physics. Springer-Verlag, New York, 1988.

4. B. Fornberg, A practical guide to pseudospectral methods. Cambridge Monographs on Applied and Computational Mathematics, 1. Cambridge University Press, Cambridge, 1996.

5. M. Funakoshi and M. OiKaWA, The resonant interaction between a long internal gravity wave and a surface gravity wave packet. J. Phys. Soc. Japan 52 (1983), no. 6, 1982-1995.

6. T. Kawahara, N. Sugimoto and T. Kakutani, Nonlinear interaction between short and long capillary-gravity waves. J. Phys. Soc. Japan 39(1975), 1379-1386.

7. Ph. C. Lauren, On a nonlinear Schrdinger equation arising in the theory of water waves. Nonlinear Anal. 24(1995), No. 4, 509-527.

8. Y. C. MA, The complete solution of the long-wave-short-wave resonance equations. Stud. Appl. Math. 59(1978), No. 3, 201-221.

9. K. Nishikawa, H. Hojo, K. Mima, and H. Ikezi, Coupled nonlinear electron-plasma and ion acoustic waves. Phys. Rev. Lett. 33(1974), 148-151.

10. A. Rashid and G. Mustafa, The Fourier pseudospectral method with restraint operator for Burgers equation. J. Univ. Sci. Technol. China 33(2003), No. 5, 547-554.

11. M. Tsutsumi, Well-posedness of the Cauchy problem for a coupled Schrdinger-KdV equation. Nonlinear mathematical problems in industry, II (Iwaki, 1992), 513-528, GAKUTO Internat. Ser. Math. Sci. Appl., 2, Gakkōtosho, Tokyo, 1993.

12. N. Yajima and M. OIKAWA, Formation and interaction of sonic Langmuir soliton. Prog. Theor. Phys. 56(1974), 1719-1739.

13. N. Yajima and J. Satsuma, Soliton solutions in a diatomic lattice system. Prog. Theor. Phys. 62(1979), 370-378.

(Received 30.11.2004)

Authors's address:

Department of Mathematics

COMSATS Institute of Information Technology

Defence Road, Off Raiwind Road

Lahore, Pakistan

E-Mail: rashid_himat@yahoo.com 